\title{
Associated Primes of Powers of Monomial Ideals: A Survey
}

\author{
Mehrdad Nasernejad \\ Department of Mathematics, Khayyam University, Mashhad, Iran \\ Email address: \\ m.nasernejad@khayyam.ac.ir
}

\section{To cite this article:}

Mehrdad Nasernejad. Associated Primes of Powers of Monomial Ideals: A Survey. International Journal of Theoretical and Applied Mathematics. Vol. 6, No. 1, 2019, pp. 1-13. doi: 10.11648/j.ijtam.20200601.11

Received: October 21, 2019; Accepted: November 12, 2019; Published: December 30, 2019

\begin{abstract}
Let $R$ be a commutative Noetherian ring and $I$ be an ideal of $R$. We say that $I$ satisfies the persistence property if $\operatorname{Ass}_{R}\left(R / I^{k}\right) \subseteq \operatorname{Ass}_{R}\left(R / I^{k+1}\right)$ for all positive integers $\mathrm{k}$, where $\operatorname{Ass}_{R}(R / I)$ denotes the set of associated prime ideals of $I$. In addition, an ideal $I$ has the strong persistence property if $\left(I^{k+1}:{ }_{R} I\right)=I^{k}$ for all positive integers $\mathrm{k}$. Also, an ideal $I$ is called normally torsion-free if $\operatorname{Ass}_{R}\left(R / I^{k}\right) \subseteq \operatorname{Ass}_{R}(R / I)$ for all positive integers $\mathrm{k}$. In this paper, we collect the latest results in associated primes of powers of monomial ideals in three concepts, i.e., the persistence property, strong persistence property, and normally torsion-freeness. Also, we present some classes of monomial ideals such that are none of edge ideals, cover ideals, and polymatroidal ideals, but satisfy the persistence property and strong persistence property. In particular, we study the Alexander dual of path ideals of unrooted starlike trees. Furthermore, we probe the normally torsion-freeness of the Alexander dual of some path ideals which are related to banana trees. We close this paper with exploring the normally torsion-freeness under some monomial operations.
\end{abstract}

Keywords: Associated Prime Ideals, Powers of Ideals, Monomial Ideals, Persistence Property, Strong Persistence Property, Normally Torsion-free

\section{Introduction}

Let $I$ be an ideal of a commutative Noetherian ring $R$. A prime ideal $\mathfrak{p} \subset R$ is an associated prime of $I$ if there exists an element $v$ in $R$ such that $\mathfrak{p}=\left(I:_{R} v\right)$, where $\left(I:_{R} v\right)=\{r \in R \mid r v \in I\}$. The set of associated primes of $I$, denoted by $\operatorname{Ass}_{R}(R / I)$, is the set of all prime ideals associated to $I$. We will be interested in the sets $\operatorname{Ass}_{R}\left(R / I^{k}\right)$ when $k$ varies. A well-known result of Brodmann [1] says that the sequence $\left\{\operatorname{Ass}_{R}\left(R / I^{k}\right)\right\}_{k \geq 1}$ of associated prime ideals is stationary for large $k$. In fact, there exists a positive integer $k_{0}$ such that $\operatorname{Ass}_{R}\left(R / I^{k}\right)=\operatorname{Ass}_{R}\left(R / I^{k_{0}}\right)$ for all integers $k \geq k_{0}$. The least such integer $k_{0}$ is called the index of stability of $I$ and $\operatorname{Ass}_{R}\left(R / I^{k_{0}}\right)$ is called the stable set of associated prime ideals to $I$, denoted by $\operatorname{Ass}^{\infty}(I)$. It should be noted that there are only a few known results providing exact calculations of the stable set and the index of stability for monomial ideals, see [2, 3, 4]. Moreover, several notions arise in the context of Brodmann's result. In this paper, we only focus on three notions, that is, persistence property, strong persistence property, and normally torsion-freeness.

In Sections 2 and 3, we will concentrate on the persistence property and strong persistence property. We say that an ideal $I$ in a commutative Noetherian ring $R$ satisfies the persistence property if $\operatorname{Ass}_{R}\left(R / I^{k}\right) \subseteq \operatorname{Ass}_{R}\left(R / I^{k+1}\right)$ for all integers $k \geq 1$. Along this argument, an ideal $I$ in a commutative Noetherian ring $R$ has the strong persistence property if $\left(I^{k+1}:_{R} I\right)=I^{k}$ for all positive integers $k$. McAdam [5] presented an example which says, in general, there exists an ideal which does not satisfy the persistence property. Now, suppose that $I$ is a monomial ideal in the polynomial ring $R=K\left[x_{1}, \ldots, x_{n}\right]$ over a field $K$ and $x_{1}, \ldots, x_{n}$ are indeterminates. It is known that there are some monomial ideals which do not satisfy the persistence property, see for counterexamples [6, 7, 8]. Furthermore, Kaiser, Stehlík, and Škrekovski [9] have shown that not all square-free monomial ideals have the persistence property. Also, Ratliff showed in [10] that $\left(I^{k+1}:_{R} I\right)=I^{k}$ for sufficient $k$. However, by applying combinatorial methods, it has been shown that many large families of square-free 
monomial ideals satisfy the persistence property and the strong persistence property. These attempts led to the persistence property and also the strong persistence property hold for edge ideals of finite simple graphs [7], edge ideals of finite graphs with loops [11], and polymatroidal ideals [12]. Furthermore, according to [13], cover ideals of perfect graphs have the persistence property. In these sections, we introduce the other classes of monomial ideals which have the persistence property or strong persistence property.

Section 4 is devoted to the study of normally torsionfreeness for monomial ideals. An ideal $I$ in a commutative Noetherian ring $R$ is called normally torsion-free if $\operatorname{Ass}_{R}\left(R / I^{k}\right) \subseteq \operatorname{Ass}_{R}(R / I)$ for all positive integers $k$. A few examples of normally torsion-free monomial ideals appear from graph theory. In [14], it has been already proved that a finite simple graph $G$ is bipartite if and only if its edge ideal is normally torsion-free. Moreover, by [15], it is well-known that the cover ideals of bipartite graphs are normally torsion-free. In addition, in [12], it has been verified that every transversal polymatroidal ideal is normally torsion-free. However, it is little known for the normally torsion-free monomial ideals which are not square-free. In this section, we express the other classes of normally torsion-free monomial ideals, and also introduce several methods for constructing new normally torsion-free non-square-free monomial ideals based on the monomial ideals which have normally torsion-freeness.

Several questions arise along these arguments for future works. In Section 5, we terminate this paper with some open questions which are devoted to the persistence property, strong persistence property, normally torsion-freeness of monomial ideals, and the unique homogeneous maximal ideal $\mathfrak{m}=$ $\left(x_{1}, \ldots, x_{n}\right)$ of $R=K\left[x_{1}, \ldots, x_{n}\right]$.

It should be noted that one can examine the persistence property for ideals in some commutative rings other than polynomial rings. To see this, one may consider two rings such that one of them is Noetherian other than the polynomial ring, say Dedekind rings, and the other one is non-Noetherian, say Prüfer domains. Recall that an integral domain $R$ is a Dedekind ring if every proper ideal in $R$ is uniquely a product of a finite number of prime ideals, see [16, Theorem 6.10]. Let $I$ be a proper ideal in a Dedekind ring $R$. Then $I$ has the persistence property ([17, Theorem 2.3$]$ ), and also it is normally torsion-free ([17, Corollary 2.5$])$. In addition, if $I$ is a non-zero ideal in a Dedekind ring $R$, then $I$ has the strong persistence property ([17, Corollary 3.2]). Remember that a Prüfer domain $R$ is an integral domain in which every nonzero finitely generated ideal is invertible. Let $I$ be a non-zero finitely generated ideal in a Prüfer domain $R$. Then, with respect to weakly associated prime ideals, one can deduce that $I$ has the persistence property ([17, Theorem 5.8]), and also it has the strong persistence property ([17, Theorem 5.2]). One may also extend the notion of the persistence property for ideals to the persistence property for rings ([17, Definition 5.9]).

Along our previous arguments, one can state the concept of the persistence property for associated primes of a family of ideals. In fact, based on [18, Definition 1.1], let $\Phi$ be a family of ideals of a commutative Noetherian ring $R$. Then we say that $\Phi$ has the persistence property if there exists a relation $\leq$ on $\Phi$ such that $(\Phi, \leq)$ is a partially ordered set with the following properties:

(i) For all $\mathfrak{b} \in \Phi$, the set $\left(\bigcup_{\mathfrak{a} \in \Phi} \operatorname{Ass}_{R}(R / \mathfrak{a})\right) \cap V(\mathfrak{b})$ is finite, where for an ideal $\mathfrak{c}$ of $R, V(\mathfrak{c})$ is the set of prime ideals contain $\mathfrak{c}$.

(ii) For all ideals $\mathfrak{a}, \mathfrak{b} \in \Phi$ with $\mathfrak{a} \leq \mathfrak{b}$, we have that $\operatorname{Ass}_{R}(R / \mathfrak{a}) \subseteq \operatorname{Ass}_{R}(R / \mathfrak{b})$.

By considering definition above, one can present two such families of ideals with the persistence property. To see one of them, let $n$ be a positive integer with $n \leq \operatorname{depth}(R)$. Put $\Phi=\left\{\left(x_{1}, \ldots, x_{n}\right): x_{1}, \ldots, x_{n}\right.$ is an $R$-regular sequence $\}$.

Then $(\Phi, \supseteq)$ satisfies the persistence property (cf. [18, Theorem 2.1]). To know another one, we refer the reader to [18, Theorem 2.3].

Throughout this paper, $R=K\left[x_{1}, \ldots, x_{n}\right]$ is the polynomial ring over a field $K$ and $x_{1}, \ldots, x_{n}$ are indeterminates. Also, for a monomial ideal $I$, we denote the unique minimal set of monomial generators of $I$ by $G(I)$. The symbol $\mathbb{N}$ (respectively, $\mathbb{N}_{0}$ ) will always denote the set of positive (respectively, non-negative) integers. Moreover, the symbol $V(G)$ (respectively, $E(G)$ ) is the set of vertices of a graph $G$ (respectively, the set of edges of a graph $G$ ) and $L_{G}$ is the set of leaves of $G$ (i.e., the set of vertices of degree one in $G$ ). We also denote the distance between two vertices $u$ and $v$ in $V(G)$ by $\mathrm{d}(u, v)$.

\section{Persistence Property for Monomial Ideals}

In this section, we present some classes of monomial ideals such that are none of edge ideals, cover ideals, and polymatroidal ideals, but satisfy the persistence property. To start our arguments, let $G$ be a finite simple graph, that is to say, $G$ has no loops and no multiple edges. The edge ideal of a graph $G$, which was introduced by Villarreal [19], is the ideal generated by the monomials $x_{i} x_{j}$, where $\{i, j\}$ is an edge of $G$. Path ideals of graphs were first introduced by Conca and De Negri [20] in the context of monomial ideals of linear type. Hereafter, one needs to recall that a path of length $t$ in a finite simple graph $G$ is a sequence of vertices $i_{1}, \ldots, i_{t+1}$ such that $e_{j}=\left\{i_{j}, i_{j+1}\right\}$ is an edge for $j=1, \ldots, t$. The path ideal corresponding to $G$ of length $t$ is defined by

$$
I_{t}(G):=\left(x_{i_{1}} \cdots x_{i_{t+1}}: i_{1}, \ldots, i_{t+1}\right.
$$

is a path of $G$ of length $t$ ), and also the Alexander dual of $I_{t}(G)$ is defined as follows

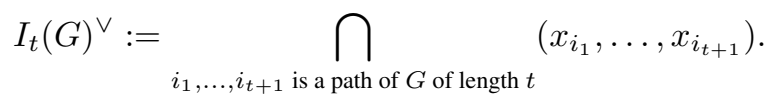

We observe that $I_{1}(G)$ is the ordinary edge ideal corresponding to $G$, and so the Alexander dual of it, i.e., 
$I_{1}(G)^{\vee}$, is exactly the cover ideal corresponding to $G$. In Theorem 2.2, we give a class of graphs, which are called centipede graphs, and show that path ideals generated by paths of length two, have the persistence property. However, chasing the proof yields that this ideal has the strong persistence property as well. In subsequent definition, we introduce the definition of centipede graphs. Definition 2.1 (see [22, Definition 2.10])The centipede graph $W_{n}$ with $n \in \mathbb{N}$, as shown in figure below, is the graph on the vertex set $\left\{a_{1}, \ldots, a_{n}\right\} \cup\left\{b_{1}, \ldots, b_{n}\right\}$. The set of edges of the centipede graph is given by

$$
E\left(W_{n}\right)=\left\{\left\{a_{i}, b_{i}\right\}: 1 \leq i \leq n\right\} \cup\left\{\left\{b_{j}, b_{j+1}\right\}: 1 \leq j \leq n-1\right\}
$$

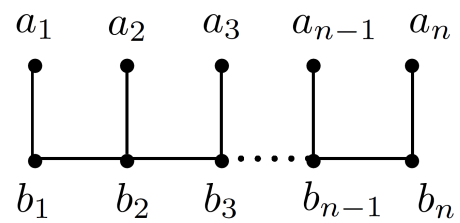

Figure 1. Centipede graph.

Theorem 2.2.(see [21, Theorem 2.11]) Let $W_{n}$, for some $n \in \mathbb{N}$ with $n \geq 2$, be a centipede graph with corresponding path ideal $I_{2}\left(W_{n}\right)$. Then $I_{2}\left(W_{n}\right)$ has the persistence property. We now turn our attention to directed graphs. To do this, we should recall some definitions from [22] which will be needed in the sequel. Definition 2.3.(see [22, Definition 2.1])A directed edge of a graph is an assignment of a direction to an edge of a graph. If $\{w, u\}$ is an edge, we write $(w, u)$ to denote the directed edge, where the direction is from $w$ to $u$. A graph is a directed graph if each edge has been assigned a direction. A path of length $t$ in a directed graph is a sequence of vertices $i_{1}, \ldots, i_{t+1}$ such that $e_{j}=\left(i_{j}, i_{j+1}\right)$ is a directed edge for $j=1, \ldots, t$. Fix a positive integer $t$ and a directed graph $G$. The path ideal of $G$ of length $t$ is the following monomial ideal $I_{t}(G):=\left(x_{i_{1}} \cdots x_{i_{t+1}}: i_{1}, \ldots, i_{t+1}\right.$ is a path of $G$ of length $\left.t\right)$, and also the Alexander dual of $I_{t}(G)$ is defined as follows:

$$
I_{t}(G)^{\vee}:=\bigcap_{i_{1}, \ldots, i_{t+1} \text { is a path of } G \text { of length } t}\left(x_{i_{1}}, \ldots, x_{i_{t+1}}\right) .
$$

A tree $T$ can be considered as a directed graph by choosing any vertex of $T$ to be the root of the tree, and assigning to each edge the direction "away" from the root. Since $T$ is a tree, the assignment of a direction will always be possible. A rooted tree $T$ is a tree with one vertex chosen as root. If tree $T$ has no such root, then we say that $T$ is unrooted.

Example 2.4. Consider

$$
T_{1}=\left(V\left(T_{1}\right), E\left(T_{1}\right)\right)
$$

and

$$
T_{2}=\left(V\left(T_{2}\right), E\left(T_{2}\right)\right)
$$

where

$$
V\left(T_{1}\right)=V\left(T_{2}\right)=\left\{v_{1}, v_{2}, v_{3}, v_{4}, v_{5}\right\}
$$

$$
E\left(T_{1}\right)=\left\{\left\{v_{1}, v_{2}\right\},\left\{v_{1}, v_{3}\right\},\left\{v_{2}, v_{4}\right\},\left\{v_{2}, v_{5}\right\}\right\},
$$

and

$$
E\left(T_{2}\right)=\left\{\left(v_{1}, v_{2}\right),\left(v_{1}, v_{3}\right),\left(v_{2}, v_{4}\right),\left(v_{2}, v_{5}\right)\right\}
$$

in the following graphs. Then the tree $T_{1}$, the left graph in figure below, is an example of a tree which is not rooted, while the tree $T_{2}$, the right graph in figure below, is rooted at the vertex $v_{1}$.
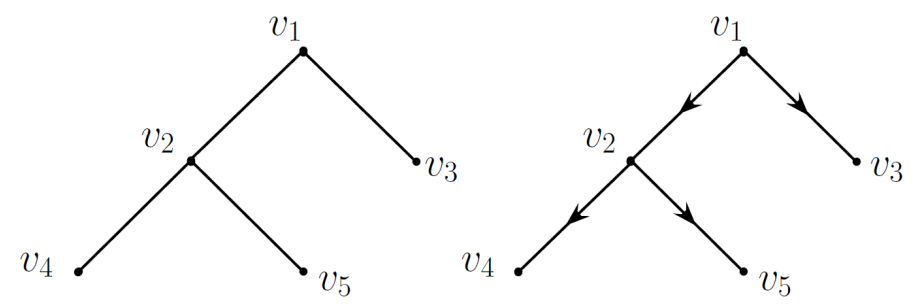

Figure 2. Unrooted and rooted trees. 
Already in [23, Theorem 3.1] (when $t=2$ ), has been proved the following theorem. Our aim is to generalize this result to unrooted symmetric starlike trees. Let $K_{1, n}$ be a star graph with the vertex set $\left\{z, x_{1}, \ldots, x_{n}\right\}$ and center $z$. Let $J_{2}$ be the corresponding Alexander dual of $I_{2}\left(K_{1, n}\right)$. Then

(1) $\left(z, x_{1}, \ldots, x_{n}\right) \in \operatorname{Ass}\left(R / J_{2}^{s}\right)$ for $s \geq n-1$ and

(2) $\left(z, x_{1}, \ldots, x_{n}\right) \notin \operatorname{Ass}\left(R / J_{2}^{s}\right)$ for $s<n-1$,

where $\left(z, x_{1}, \ldots, x_{n}\right) \subset K\left[z, x_{1}, \ldots, x_{n}\right]$ is the maximal ideal.

To understand Theorem 2.8, one requires to know the definition of starlike graphs. To do this, we begin with the following definition. Definition 2.6. (see [22, Definition 2.4]) A tree is said to be starlike if exactly one of its vertices has degree greater than two. This vertex is called the center of the starlike.

We also say that $T$ is a symmetric starlike tree if $T$ is a starlike tree and $\mathrm{d}(u, v)=\mathrm{d}\left(u^{\prime}, v^{\prime}\right)$ for all $u, u^{\prime}, v, v^{\prime} \in L_{T}$ with $u \neq v$ and $u^{\prime} \neq v^{\prime}$.

Example 2.7. Consider the following trees. Then the left graph in figure below is an example of a starlike tree with center $z$, while the right graph in figure below is an example of a symmetric starlike tree with center $z$.
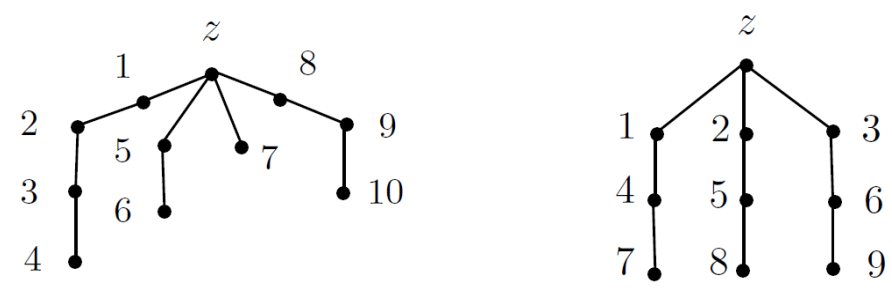

Figure 3. Starlike and symmetric starlike trees.

In the sequel, we probe the persistence property for the Alexander dual of path ideals of unrooted starlike trees.

Theorem 2.8.(see [22, Theorem 2.6]) Let $T$ be an unrooted starlike tree on the vertex set $\{z, 1, \ldots, n\}$ with center $z$. Also let $I$ be the monomial ideal corresponding to $T$ which is generated by the paths of maximal lengths, and corresponding Alexander dual $J$. Then the ideal $J$ has the persistence property.

In the next example we clarify the main goal of Theorem 2.8. Example 2.9.(see [22, Example 2.7])Suppose that $T$ is the unrooted starlike tree with center $\mathrm{z}$, as the left graph in Figure 3. By using the notations that we used in Theorem 2.8, we have

$$
\begin{aligned}
I= & \left(x_{4} x_{3} x_{2} x_{1} x_{z} x_{8} x_{9} x_{10}, x_{4} x_{3} x_{2} x_{1} x_{z} x_{7}, x_{5} x_{6} x_{z} x_{7}, x_{4} x_{3} x_{2} x_{1} x_{z} x_{5} x_{6},\right. \\
& \left.x_{5} x_{6} x_{z} x_{8} x_{9} x_{10}, x_{7} x_{z} x_{8} x_{9} x_{10}\right),
\end{aligned}
$$

and so

$$
\begin{array}{r}
J=\left(x_{4}, x_{3}, x_{2}, x_{1}, x_{z}, x_{8}, x_{9}, x_{10}\right) \cap\left(x_{4}, x_{3}, x_{2}, x_{1}, x_{z}, x_{7}\right) \cap\left(x_{5}, x_{6}, x_{z}, x_{7}\right) \\
\cap\left(x_{4}, x_{3}, x_{2}, x_{1}, x_{z}, x_{5}, x_{6}\right) \cap\left(x_{5}, x_{6}, x_{z}, x_{8}, x_{9}, x_{10}\right) \cap\left(x_{7}, x_{z}, x_{8}, x_{9}, x_{10}\right) .
\end{array}
$$

According to Theorem 2.8, the ideal $J$ has the persistence property. The corollary below is an immediate consequence of Theorem 2.8.
Corollary 2.10.(see [22, Corollary 2.8]) Suppose that $T$ is an unrooted symmetric starlike tree on the vertex set $V(T)=$ $\{z, 1, \ldots, n\}$ with center $z$ and the following edge set.

$$
E(T)=\{\{z, i\},\{k j+i, k j+k+i\} \mid i=1, \ldots, k, j=0, \ldots, m-1\},
$$

Such that $n=k(m+1)$ for some $k \in \mathbb{N}$ and $m \in \mathbb{N}_{0}$. Suppose also that $I_{2 m+2}(T)$ is the path ideal corresponding to $T$ of length $2 m+2$ and corresponding Alexander dual $J_{2 m+2}$. Then the ideal $J_{2 m+2}$ has the persistence property.
Let us illustrate Corollary 2.10 with an example. Example 2.11. Suppose that $T$ is the unrooted symmetric starlike tree with center $z$, as shown in Figure 3, on the vertex set $V(T)=$ $\{z, 1,2,3,4,5,6,7,8,9\}$. Then

$$
I_{6}(T)=\left(x_{7} x_{4} x_{1} x_{z} x_{2} x_{5} x_{8}, x_{7} x_{4} x_{1} x_{z} x_{3} x_{6} x_{9}, x_{8} x_{5} x_{2} x_{z} x_{3} x_{6} x_{9}\right) .
$$


It follows that the Alexander dual of $I_{6}(T)$, i.e. $J_{6}$, is given by

$$
\begin{aligned}
& J_{6}=\left(x_{7}, x_{4}, x_{1}, x_{z}, x_{2}, x_{5}, x_{8}\right) \cap\left(x_{7}, x_{4}, x_{1}, x_{z}, x_{3}, x_{6}, x_{9}\right) \cap\left(x_{8}, x_{5}, x_{2}, x_{z}, x_{3}, x_{6}, x_{9}\right) \\
& \quad=\left(x_{z}, x_{8} x_{9}, x_{7} x_{9}, x_{5} x_{9}, x_{4} x_{9}, x_{2} x_{9}, x_{1} x_{9}, x_{7} x_{8}, x_{6} x_{8}, x_{4} x_{8}, x_{3} x_{8}, x_{1} x_{8}, x_{6} x_{7},\right. \\
& \quad x_{5} x_{7}, x_{3} x_{7}, x_{2} x_{7}, x_{5} x_{6}, x_{4} x_{6}, x_{2} x_{6}, x_{1} x_{6}, x_{4} x_{5}, x_{3} x_{5}, x_{1} x_{5}, x_{3} x_{4}, x_{2} x_{4}, x_{2} x_{3}, \\
& \left.\quad x_{1} x_{3}, x_{1} x_{2}\right) .
\end{aligned}
$$

Now, Corollary 2.10 implies that the ideal $J_{6}$ has the persistence property.

We now examine when the unique homogenous maximal ideal appears. To achieve this, we first recall the definition of the expansion operator on monomial ideals which has been stated in [24], and then apply it as a criterion for the persistence property of monomial ideals.

Let $R$ be the polynomial ring over a field $K$ in the variables $x_{1}, \ldots, x_{n}$. Fix an ordered $n$-tuple $\left(i_{1}, \ldots, i_{n}\right)$ of positive integers, and consider the polynomial $\operatorname{ring} R^{\left(i_{1}, \ldots, i_{n}\right)}$ over $K$ in the variables

$$
x_{11}, \ldots, x_{1 i_{1}}, x_{21}, \ldots, x_{2 i_{2}}, \ldots, x_{n 1}, \ldots, x_{n i_{n}} .
$$

Let $\mathfrak{p}_{j}$ be the monomial prime ideal $\left(x_{j 1}, x_{j 2}, \ldots, x_{j i_{j}}\right) \subseteq$ $R^{\left(i_{1}, \ldots, i_{n}\right)}$ for all $j=1, \ldots, n$. Attached to each monomial ideal $I \subset R$ with a set of monomial generators $\left\{\mathbf{x}^{\mathbf{a}_{1}}, \ldots, \mathbf{x}^{\mathbf{a}_{m}}\right\}$, where $\mathbf{x}^{\mathbf{a}_{i}}=x_{1}{ }^{a_{i}(1)} \cdots x_{n}{ }^{a_{i}(n)}$ and $a_{i}(j)$ denotes the $j$-th component of the vector $\mathbf{a}_{i}=$ $\left(a_{i}(1), \ldots, a_{i}(n)\right)$ for all $i=1, \ldots, m$. We define the expansion of I with respect to the $n$-tuple $\left(i_{1}, \ldots, i_{n}\right)$, denoted by $I^{\left(i_{1}, \ldots, i_{n}\right)}$, to be the monomial ideal

$$
I^{\left(i_{1}, \ldots, i_{n}\right)}=\sum_{i=1}^{m} \mathfrak{p}_{1}^{a_{i}(1)} \cdots \mathfrak{p}_{n}^{a_{i}(n)} \subseteq R^{\left(i_{1}, \ldots, i_{n}\right)} .
$$

We simply write $R^{*}$ and $I^{*}$, respectively, rather than $R^{\left(i_{1}, \ldots, i_{n}\right)}$ and $I^{\left(i_{1}, \ldots, i_{n}\right)}$.

Example 2.12. Consider $R=K\left[x_{1}, x_{2}, x_{3}\right]$ and the ordered 3-tuple $(1,3,2)$. Then we have $\mathfrak{p}_{1}=\left(x_{11}\right)$, $\mathfrak{p}_{2}=\left(x_{21}, x_{22}, x_{23}\right)$, and $\mathfrak{p}_{3}=\left(x_{31}, x_{32}\right)$. So for the monomial ideal $I=\left(x_{1} x_{2}, x_{3}^{2}\right)$, the ideal $I^{*} \subseteq$ $K\left[x_{11}, x_{21}, x_{22}, x_{23}, x_{31}, x_{32}\right]$ is $\mathfrak{p}_{1} \mathfrak{p}_{2}+\mathfrak{p}_{3}^{2}$, namely

$$
I^{*}=\left(x_{11} x_{21}, x_{11} x_{22}, x_{11} x_{23}, x_{31}^{2}, x_{31} x_{32}, x_{32}^{2}\right) .
$$

In order to prove Theorem 2.14 below, one needs the following lemma.

Lemma 2.13.(see [22, Lemma 2.9]) Let I be a monomial ideal of $R$. Then I has the persistence property if and only if $I^{*}$ has.

We are now in a position to verify Theorem 2.14 .

$$
\bigcap_{i, j \in\{1, \ldots, k\}, i \neq j}\left(\mathfrak{p}_{i}+\mathfrak{p}_{j}\right)=\sum_{j=1}^{k} \mathfrak{p}_{1} \cap \cdots \cap \mathfrak{p}_{j-1} \cap \widehat{\mathfrak{p}}_{j} \cap \mathfrak{p}_{j+1} \cap \cdots \cap \mathfrak{p}_{k},
$$

Where $\widehat{\mathfrak{p}_{j}}$ means that this term is omitted. Due to $\mathfrak{p}_{i}$ and $\mathfrak{p}_{j}$, for every $i, j \in\{1, \ldots, k\}$ with $i \neq j$, are generated by
Theorem 2.14.(see [22, Theorem 2.10]) Let $T$ be an unrooted starlike tree on the vertex set $\{z, 1, \ldots, n\}$ with center $z$. Also let I be the monomial ideal corresponding to $T$ which is generated by the paths of maximal lengths, and corresponding Alexander dual J. If $\operatorname{deg}_{T} z=k$, then

$$
\begin{aligned}
& \text { (1) }\left(x_{z}, x_{1}, \ldots, x_{n}\right) \in \operatorname{Ass}_{R^{\prime}}\left(R^{\prime} / J^{s}\right) \text { for } s \geq k-1 \text {, and } \\
& \text { (2) }\left(x_{z}, x_{1}, \ldots, x_{n}\right) \notin \operatorname{Ass}_{R^{\prime}}\left(R^{\prime} / J^{s}\right) \text { for } s<k-1,
\end{aligned}
$$

where $\left(x_{z}, x_{1}, \ldots, x_{n}\right)$ is the unique homogeneous maximal ideal in the polynomial ring $R^{\prime}=K\left[x_{z}, x_{1}, \ldots, x_{n}\right]$.

Proof We give a sketch of the proof. Since $\operatorname{deg}_{T} z=$ $k$, the graph $T \backslash\{z\}$ has exactly $k$ connected components, say $L_{1}, \ldots, L_{k}$, where each component is a line graph with $\left|V\left(L_{i}\right)\right|=h_{i}$ for each $i=1, \ldots, k$. Put $h_{0}:=0$ and for $i=1, \ldots, k$, let

$$
V\left(L_{i}\right):=\left\{h_{1}+\cdots+h_{i-1}+j: j=1, \ldots, h_{i}\right\} .
$$

This implies that $E(T)$ is given by

$$
\begin{array}{r}
\left\{\left\{z, \sum_{t=1}^{i-1} h_{t}+1\right\},\left\{\sum_{t=1}^{i-1} h_{t}+j, \sum_{t=1}^{i-1} h_{t}+j+1\right\}:\right. \\
\left.i=1, \ldots, k, j=1, \ldots, h_{i}-1\right\} .
\end{array}
$$

Now, set

$$
\begin{array}{r}
\mathfrak{p}_{i}:=\left(x_{h_{1}+\cdots+h_{i-1}+j}, x_{h_{1}+\cdots+h_{i-1}+j+1}:\right. \\
\left.j=1, \ldots, h_{i}-1\right)
\end{array}
$$

for $i=1, \ldots, k$. Then one can conclude that

$$
J=\bigcap_{i, j \in\{1, \ldots, k\}, i \neq j}\left(\mathfrak{p}_{i}+x_{z} R^{\prime}+\mathfrak{p}_{j}\right),
$$

and so

$$
J=x_{z} R^{\prime}+\bigcap_{i, j \in\{1, \ldots, k\}, i \neq j}\left(\mathfrak{p}_{i}+\mathfrak{p}_{j}\right)
$$

It is routine to check that

$$
J=x_{z} R^{\prime}+\sum_{j=1}^{k} \mathfrak{p}_{1} \cdots \mathfrak{p}_{j-1} \widehat{\mathfrak{p}_{j}} \mathfrak{p}_{j+1} \cdots \mathfrak{p}_{k}
$$


Now, consider the following monomial ideal

$$
\mathfrak{a}:=x_{z} R^{\prime}+\sum_{j=1}^{k}\left(x_{1} \cdots x_{j-1} \widehat{x_{j}} x_{j+1} \cdots x_{k}\right) R^{\prime},
$$

where $\widehat{x_{j}}$ means that this term is omitted. In order to use Lemma 2.13, set $\mathfrak{p}_{k+1}:=x_{z} R^{\prime}$. This implies that $J$ is the expansion of $\mathfrak{a}$ with respect to the $(k+1)$-tuple $\left(h_{1}, h_{2}, h_{3}, \ldots, h_{k}, 1\right)$. Accoridng to [23, Lemma 2.5], when $t=2$, we obtain $\mathfrak{a}=J_{2}(G)$, where $G=K_{1, n}$ is the star graph on the vertex set $\{z, 1, \ldots, n\}$ with center $z$ and corresponding Alexander dual $J_{2}(G)$. Therefore the result follows immediately from Lemma 2.13, [24, Proposition 1.2], and Theorem 2.5.

We can apply Theorem 2.14 to generalize Theorem to unrooted symmetric starlike trees in the following corollary.

Corollary 2.15.(see [22, Corollary 2.11]) Suppose that $T$ is an unrooted symmetric starlike tree on the vertex set $V(T)=$ $\{z, 1, \ldots, n\}$ with center $z$ and the following edge set

$$
\begin{gathered}
E(T)=\{\{z, i\},\{k j+i, k j+k+i\} \mid \\
i=1, \ldots, k \text { and } j=0, \ldots, m-1\}
\end{gathered}
$$

such that $n=k(m+1)$ for some $k \in \mathbb{N}$ and $m \in \mathbb{N}_{0}$. Suppose also that $I_{2 m+2}(T)$ is the path ideal corresponding to $T$ of length $2 m+2$ and corresponding Alexander dual $J_{2 m+2}$. Then

(1) $\left(x_{z}, x_{1}, \ldots, x_{n}\right) \in \operatorname{Ass}_{R^{\prime}}\left(R^{\prime} / J_{2 m+2}^{s}\right)$ for $s \geq k-1$, and

(2) $\left(x_{z}, x_{1}, \ldots, x_{n}\right) \notin \operatorname{Ass}_{R^{\prime}}\left(R^{\prime} / J_{2 m+2}^{s}\right)$ for $s<k-1$,

where $\left(x_{z}, x_{1}, \ldots, x_{n}\right)$ is the unique homogeneous maximal ideal in the polynomial ring $R^{\prime}=K\left[x_{z}, x_{1}, \ldots, x_{n}\right]$. The subsequent example illuminates what happens in Corollary 2.15 .

Example 2.16. (see [22, Example 2.12]) Suppose that $T$ is the unrooted symmetric starlike tree, as shown in figure below, on the following vertex set

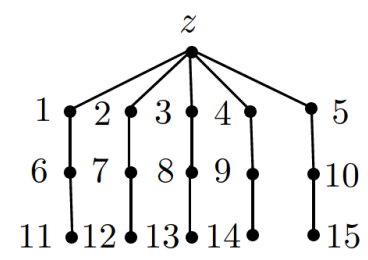

Figure 4. Unrooted symmetric starlike tree T.

Hence we obtain the path ideal $I_{6}(T)$, which is given by

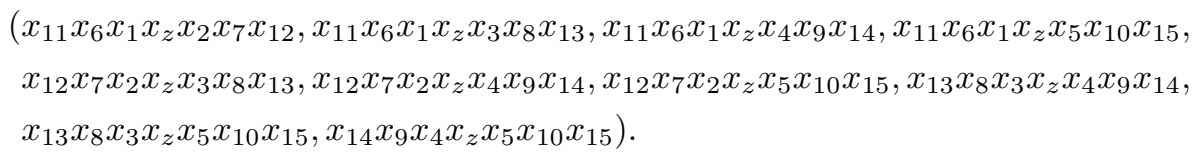

It follows that the Alexander dual of $I_{6}(T)$ is given by

$$
\begin{aligned}
J_{6} & =\left(x_{11}, x_{6}, x_{1}, x_{z}, x_{2}, x_{7}, x_{12}\right) \cap\left(x_{11}, x_{6}, x_{1}, x_{z}, x_{3}, x_{8}, x_{13}\right) \\
& \cap\left(x_{11}, x_{6}, x_{1}, x_{z}, x_{4}, x_{9}, x_{14}\right) \cap\left(x_{11}, x_{6}, x_{1}, x_{z}, x_{5}, x_{10}, x_{15}\right) \\
& \cap\left(x_{12}, x_{7}, x_{2}, x_{z}, x_{3}, x_{8}, x_{13}\right) \cap\left(x_{12}, x_{7}, x_{2}, x_{z}, x_{4}, x_{9}, x_{14}\right) \\
& \cap\left(x_{12}, x_{7}, x_{2}, x_{z}, x_{5}, x_{10}, x_{15}\right) \cap\left(x_{13}, x_{8}, x_{3}, x_{z}, x_{4}, x_{9}, x_{14}\right) \\
& \cap\left(x_{13}, x_{8}, x_{3}, x_{z}, x_{5}, x_{10}, x_{15}\right) \cap\left(x_{14}, x_{9}, x_{4}, x_{z}, x_{5}, x_{10}, x_{15}\right) .
\end{aligned}
$$

Due to Theorem 2.14, one can conlcude that

(1) $\left(x_{z}, x_{1}, \ldots, x_{15}\right) \in \operatorname{Ass}_{R^{\prime}}\left(R^{\prime} / J_{6}^{s}\right)$ for $s \geq 4$, and

(2) $\left(x_{z}, x_{1}, \ldots, x_{15}\right) \notin \operatorname{Ass}_{R^{\prime}}\left(R^{\prime} / J_{6}^{s}\right)$ for $s<4$,

Where $\left(x_{z}, x_{1}, \ldots, x_{15}\right)$ is the unique homogeneous maximal ideal in the polynomial ring $R^{\prime}=$ $K\left[x_{z}, x_{1}, \ldots, x_{15}\right]$.

The example below illuminates our method for constructing new monomial ideals which have the persistence property based on the monomial ideals so that they have the persistence property. To achieve this, one requires the following proposition.

Proposition 2.17.(see [25, Proposition 4.4]) Let I be a monomial ideal in a polynomial ring $R=K\left[x_{1}, \ldots, x_{n}\right]$ which has the persistence property, and let $u=x_{i_{1}}^{a_{1}} \cdots x_{i_{r}}^{a_{r}}$ be a monomial in $R$ with $a_{1}, \ldots, a_{r} \in \mathbb{N}$. Then $u I$ has the persistence property.

Example 2.18.(see [25, Example 4.5]) Let the monomial ideal 


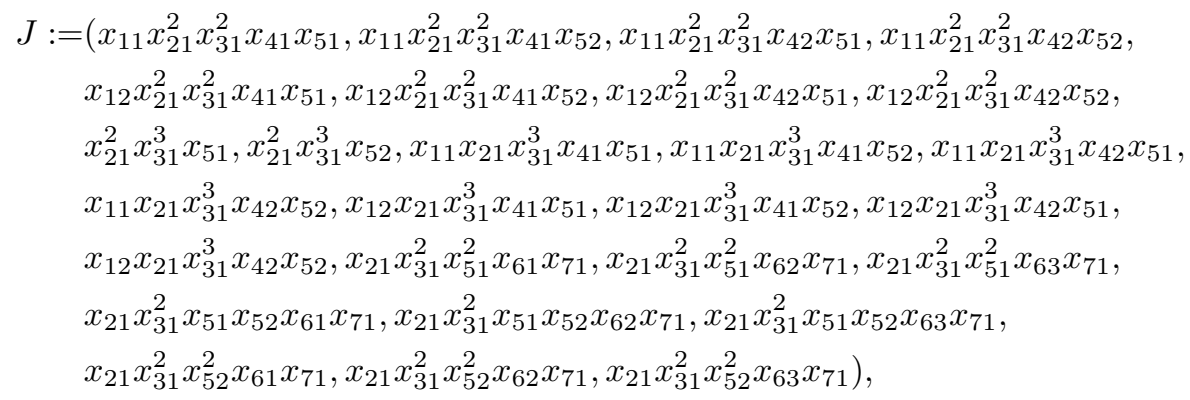

$\mathrm{Be}$ in the polynomial ring

$R=K\left[x_{11}, x_{12}, x_{21}, x_{31}, x_{41}, x_{42}, x_{51}, x_{52}, x_{61}, x_{62}, x_{63}, x_{71}\right]$

We claim that $J$ has the persistence property. To do this, put $\mathfrak{p}_{1}:=\left(x_{11}, x_{12}\right), \mathfrak{p}_{2}:=\left(x_{21}\right), \mathfrak{p}_{3}:=\left(x_{31}\right), \mathfrak{p}_{4}:=\left(x_{41}, x_{42}\right)$, $\mathfrak{p}_{5}:=\left(x_{51}, x_{52}\right), \mathfrak{p}_{6}:=\left(x_{61}, x_{62}, x_{63}\right)$, and $\mathfrak{p}_{7}:=\left(x_{71}\right)$. It is routine to check that

$$
J=\mathfrak{p}_{1} \mathfrak{p}_{2}^{2} \mathfrak{p}_{3}^{2} \mathfrak{p}_{4} \mathfrak{p}_{5}+\mathfrak{p}_{2}^{2} \mathfrak{p}_{3}^{3} \mathfrak{p}_{5}+\mathfrak{p}_{1} \mathfrak{p}_{2} \mathfrak{p}_{3}^{3} \mathfrak{p}_{4} \mathfrak{p}_{5}+\mathfrak{p}_{2} \mathfrak{p}_{3}^{2} \mathfrak{p}_{5}^{2} \mathfrak{p}_{6} \mathfrak{p}_{7}
$$

Now, consider the following monomial ideal

$$
I:=\left(x_{1} x_{2}^{2} x_{3}^{2} x_{4} x_{5}, x_{2}^{2} x_{3}^{3} x_{5}, x_{1} x_{2} x_{3}^{3}, x_{4} x_{5}, x_{2} x_{3}^{2} x_{5}^{2} x_{6} x_{7}\right),
$$

in the polynomial ring $R_{1}=K\left[x_{1}, x_{2}, x_{3}, x_{4}, x_{5}, x_{6}, x_{7}\right]$. It is easy to see that $J$ is the expansion of $I$, with respect to the 7 -tuple $(2,1,1,2,2,3,1)$. In order to prove our claim, set $I_{1}:=\left(x_{1} x_{2} x_{4}, x_{2} x_{3}, x_{1} x_{3} x_{4}, x_{5} x_{6} x_{7}\right)$ and $u:=x_{2} x_{3}^{2} x_{5}$. Based on Definition 3, the ideal $I_{1}$ is a unisplit monomial ideal. Theorems 3 and 3 imply that $I_{1}$ has the persistence property and, by Proposition 2.17, one can conclude that $I=u I_{1}$ has the persistence property. Now, Lemma 2.13 implies that $J$ has the persistence property, as required.

We end up this section by stating a theorem which yields a necessary and sufficient condition whether the unique homogeneous maximal ideal appears in the set of associated prime ideals.

Theorem 2.19.(see [26, Theorem 2.7]) Suppose that I is a monomial ideal in a polynomial ring $R=K\left[x_{1}, \ldots, x_{n}\right]$, $\mathfrak{m}=\left(x_{1}, \ldots, x_{n}\right)$, and

$$
G(I)=\left\{x_{1}^{r_{1,1}} \cdots x_{n}^{r_{1, n}}, \ldots, x_{1}^{r_{k, 1}} \cdots x_{n}^{r_{k, n}}\right\},
$$

it with $k \geq n$. Then $\mathfrak{m} \in \operatorname{Ass}_{R}(R / I)$ if and only if there exist distinct integers $i_{1}, \ldots, i_{n} \in\{1, \ldots, k\}$ such that the following conditions hold:

(i) $\left|C_{j}\right|=1$, where $C_{j}=\left\{i_{t} \mid r_{i_{t}, j}=\right.$ $\left.\max \left\{r_{i_{1}, j}, \ldots, r_{i_{n}, j}\right\}\right\}$ for all $j=1, \ldots, n$;

(ii) $C_{i} \cap C_{j}=\emptyset$ for all $i \neq j$;

(iii) $x_{1}^{r_{i, 1}} \cdots x_{n}^{r_{i, n}} \nmid x_{1}^{r_{i_{1}, 1}-1} \cdots x_{n}^{r_{i_{n}, n}-1}$ for each $i \in$ $\{1, \ldots, k\} \backslash\left\{i_{1}, \ldots, i_{n}\right\}$.

We are now ready to present an example which illustrates the details of Theorem 2.19.
It Example 2.20. (see [26, Example 2.8]) Consider the following monomial ideal

$$
I=\left(x_{1}^{2} x_{2}^{4} x_{3}, x_{1}^{3} x_{2}^{3} x_{3}, x_{1}^{4} x_{2}^{2} x_{3}, x_{1} x_{2}^{3} x_{3}^{4}, x_{1}^{3} x_{2}^{2} x_{3}^{3}\right),
$$

in the polynomial ring $R=K\left[x_{1}, x_{2}, x_{3}\right]$. Our purpose is to demonstrate that $\mathfrak{m}=\left(x_{1}, x_{2}, x_{3}\right) \in \operatorname{Ass}_{R}(R / I)$. To achieve this, it is sufficient to apply Theorem 2.19. To see this, set $u_{1}:=x_{1}^{2} x_{2}^{4} x_{3}, u_{2}:=x_{1}^{3} x_{2}^{3} x_{3}, u_{3}:=x_{1}^{4} x_{2}^{2} x_{3}, u_{4}:=x_{1} x_{2}^{3} x_{3}^{4}$, and $u_{5}:=x_{1}^{3} x_{2}^{2} x_{3}^{3}$. By choosing $i_{1}=1, i_{2}=4$, and $i_{3}=5$, one has $C_{1}=\{5\}, C_{2}=\{1\}$, and $C_{3}=\{4\}$. This implies that $\left|C_{1}\right|=\left|C_{2}\right|=\left|C_{3}\right|=1$, and also $C_{i} \cap C_{j}=\emptyset$ for all $i \neq j$, that is, the conditions (i) and (ii) are proved. Moreover, since $r_{5,1}=3, r_{1,2}=4$, and $r_{4,3}=4$, and by virtue of $u_{i} \nmid x_{1}^{2} x_{2}^{3} x_{3}^{3}$ for each $i \in\{1,2,3,4,5\} \backslash\{1,4,5\}$, one can conclude that the condition (iii) holds. Therefore, $\mathfrak{m}=$ $\left(x_{1}, x_{2}, x_{3}\right) \in \operatorname{Ass}_{R}(R / I)$. In general, there are $\left(\begin{array}{l}5 \\ 3\end{array}\right)=10$ cases for choosing $i_{1}, i_{2}$, and $i_{3}$. Direct computations show that we can also consider the following cases:

(1) $i_{1}=1, i_{2}=2$, and $i_{3}=4$.

(2) $i_{1}=2, i_{2}=3$, and $i_{3}=5$.

\section{Strong Persistence Property for Monomial Ideals}

Recall that an ideal $I$ in a commutative Noetherian ring $S$ has the strong persistence property if $\left(I^{k+1}:_{S} I\right)=I^{k}$ for all $k \in \mathbb{N}$. It is important to observe that if $I$ has the strong persistence property, then $I$ has the persistence property, see [21, Proposition 2.9] for more details. On the other hand, there exist some monomial ideals which have the persistence property, but do not necessary have the strong persistence property. As an example, consider the ideal $I$ generated by monomials $x_{1} x_{2} x_{3}, x_{1} x_{2} x_{4}, x_{1} x_{3} x_{5}, x_{1} x_{4} x_{6}$, $x_{1} x_{5} x_{6}, x_{2} x_{3} x_{6}, x_{2} x_{4} x_{5}, x_{2} x_{5} x_{6}, x_{3} x_{4} x_{5}$, and $x_{3} x_{4} x_{6}$ in the polynomial ring $R=K\left[x_{1}, x_{2}, x_{3}, x_{4}, x_{5}, x_{6}\right]$. It follows now from [7, Example 2.18] that $I$ has the persistence property. Furthermore, direct computation implies that $\left(I^{3}:_{R} I\right) \neq I^{2}$, and so $I$ has no strong persistence property.

In this section, we give some classes of monomial ideals which satisfy the strong persistence property. To accomplish this, we begin with the following definition.

Definition 3.1.(see [25, Definition 2.1]) Let $I$ be a monomial ideal in the polynomial ring $R=K\left[x_{1}, \ldots, x_{n}\right]$ with 
the unique minimal set of monomial generators $G(I)=$ $\left\{u_{1}, \ldots, u_{m}\right\}$. Then we say that $I$ is a unisplit monomial ideal if there exists $i \in \mathbb{N}$ with $1 \leq i \leq m$ such that each monomial $u_{j}$ has no common factor with $u_{i}$ for all $j \in \mathbb{N}$ with $1 \leq j \leq m$ and $j \neq i$. We call $u_{i}$ as split generator.

Example 3.2.(see [25, Example 2.2]) Consider the monomial ideal

$$
I=\left(x_{3}^{2} x_{5} x_{6}^{3}, x_{1}^{3} x_{2}^{2} x_{4}^{4}, x_{1}^{6} x_{2}^{3} x_{7}^{4}, x_{2}^{2} x_{7}^{4} x_{4}^{5}\right),
$$

in the polynomial ring $R=K\left[x_{1}, x_{2}, x_{3}, x_{4}, x_{5}, x_{6}, x_{7}\right]$. It is easy to see that $I$ is a unisplit monomial ideal of $R$ with $x_{3}^{2} x_{5} x_{6}^{3}$ is the split generator.

The following notion of separable monomial ideals is needed in the sequel.

Definition 3.3.(see [25, Definition 2.3]) Let $I$ be a monomial ideal in the polynomial ring $R=K\left[x_{1}, \ldots, x_{n}\right]$ with the unique minimal set of monomial generators $G(I)=$ $\left\{u_{1}, \ldots, u_{m}\right\}$. Then we say that $I$ is a separable monomial ideal if there exist $i \in \mathbb{N}$ with $1 \leq i \leq m$ and monomials $g$ and $w$ in $R$ such that $w \neq 1, u_{i}=w g, \operatorname{gcd}(w, g)=1$, and for all $j \in \mathbb{N}$ with $1 \leq j \neq i \leq m, \operatorname{gcd}\left(u_{j}, u_{i}\right)=w$.

Example 3.4.(see [25, Example 2.4]) Consider the monomial ideal

$$
I=\left(x_{1} x_{2} x_{3}^{3} x_{4}^{5}, x_{1}^{2} x_{2}^{3} x_{3}^{4} x_{5}^{3} x_{6}^{5}, x_{1}^{2} x_{2} x_{3}^{5} x_{5}^{2} x_{6}, x_{1}^{3} x_{2}^{2} x_{3}^{6} x_{6}^{4}\right),
$$

in the polynomial ring $R=K\left[x_{1}, x_{2}, x_{3}, x_{4}, x_{5}, x_{6}\right]$. Then, by setting

$$
\begin{aligned}
& u_{1}:=x_{1} x_{2} x_{3}^{3} x_{4}^{5}, u_{2}:=x_{1}^{2} x_{2}^{3} x_{3}^{4} x_{5}^{3} x_{6}^{5}, \\
& u_{3}:=x_{1}^{2} x_{2} x_{3}^{5} x_{5}^{2} x_{6}, u_{4}:=x_{1}^{3} x_{2}^{2} x_{3}^{6} x_{6}^{4},
\end{aligned}
$$

$i:=1$ and $w:=x_{1} x_{2} x_{3}^{3}$, one can easily check that $I$ is a separable monomial ideal of $R$.

Note that the set of unisplit monomial ideals and the set of separable monomial ideals are disjoint.

In the sequel, we first state condition $(\sharp)$ on monomial ideals, and we next emphasize that any monomial ideal that satisfying condition $(\sharp)$ has the strong persistence property.

Definition 3.5.(see [25, Definition 2.7]) Suppose that $I$ is a monomial ideal in the polynomial ring $R=K\left[x_{1}, \ldots, x_{n}\right]$ with $G(I)=\left\{u_{1}, \ldots, u_{m}\right\}$. We say that $I$ satisfies condition $(\sharp)$ if there exists a positive integer $i$ with $1 \leq i \leq m$ such that

$$
\begin{gathered}
\left(u_{1}^{\alpha_{1}} \cdots u_{i-1}^{\alpha_{i-1}} \widehat{u_{i}^{\alpha_{i}}} u_{i+1}^{\alpha_{i+1}} \cdots u_{m}^{\alpha_{m}} u_{j}:_{R} u_{i}\right)= \\
u_{1}^{\alpha_{1}} \cdots u_{i-1}^{\alpha_{i-1}} \widehat{u_{i}^{\alpha_{i}}} u_{i+1}^{\alpha_{i+1}} \cdots u_{m}^{\alpha_{m}}\left(u_{j}:_{R} u_{i}\right)
\end{gathered}
$$

for all $j=1, \ldots, m$ with $j \neq i$ and $\alpha_{1}, \ldots, \alpha_{m} \in \mathbb{N}_{0}$, where $\widehat{u_{i}^{\alpha_{i}}}$ means that this term is omitted and $\mathbb{N}_{0}$ is the set of nonnegative integers.

The following theorems characterize any monomial ideal that satisfying condition $(\sharp)$.

Theorem 3.6. (see [25, Theorem 2.9]) Let I be an ideal satisfies condition ( $\sharp)$. Then $I$ is either a unisplit monomial ideal or a separable monomial ideal.

Theorem 3.7.(see [25, Theorem 2.10]) Every unisplit monomial ideal of $R=K\left[x_{1}, \ldots, x_{n}\right]$ satisfies condition $(\sharp)$.
Theorem 3.8. (see [25, Theorem 2.11]) Every separable monomial ideal of $R=K\left[x_{1}, \ldots, x_{n}\right]$ satisfies condition $(\sharp)$.

Here, we describe the relation between condition $(\sharp)$ and the strong persistence property in theorem below.

Theorem 3.9.(see [25, Theorem 3.1]) Let $J$ be a monomial ideal satisfies condition $(\sharp)$. We then have $\left(J^{k+1}:_{R} J\right)=J^{k}$ for all $k \in \mathbb{N}$, i.e., $J$ has the strong persistence property.

Proof. We will sketch the proof. Without loss of generality, suppose that $G(J)=\left\{u_{1}, \ldots, u_{m}\right\}$ is the unique minimal set of monomial generators of $J$ such that

$$
\left(u_{2}^{\alpha_{2}} \cdots u_{m}^{\alpha_{m}} u_{j}:_{R} u_{1}\right)=u_{2}^{\alpha_{2}} \cdots u_{m}^{\alpha_{m}}\left(u_{j}:_{R} u_{1}\right)
$$

for all $j=2, \ldots, m$ and $\alpha_{2}, \ldots, \alpha_{m} \in \mathbb{N}_{0}$. We need only show that $\left(J^{k+1}:_{R} J\right) \subseteq J^{k}$ for all $k \in \mathbb{N}$. Fix $k \in \mathbb{N}$. As $J=\sum_{j=1}^{m} u_{j} R$, this implies that

$$
\left(J^{k+1}:_{R} J\right)=\bigcap_{j=1}^{m}\left(J^{k+1}:_{R} u_{j}\right)
$$

Note also that $J^{k+1}=J^{k}\left(\sum_{i=1}^{m} u_{i} R\right)=\sum_{i=1}^{m} J^{k} u_{i}$. It follows that

$$
\left(J^{k+1}:_{R} J\right)=\bigcap_{j=1}^{m} \sum_{i=1}^{m}\left(J^{k} u_{i}:_{R} u_{j}\right) .
$$

On the other hand, one can conclude that

$$
\sum_{i=1}^{m}\left(J^{k} u_{i}:_{R} u_{1}\right)=J^{k}+\sum_{i=2}^{m}\left(J^{k} u_{i}:_{R} u_{1}\right)
$$

However, for $i \in \mathbb{N}$ with $2 \leq i \leq m$, we have the following equalities

$$
\begin{gathered}
\left(J^{k} u_{i}:_{R} u_{1}\right)=\sum_{\alpha_{1}+\cdots+\alpha_{m}=k, \alpha_{1}>0}\left(u_{1}^{\alpha_{1}} u_{2}^{\alpha_{2}} \cdots u_{m}^{\alpha_{m}} u_{i}:_{R} u_{1}\right) \\
+\sum_{\alpha_{2}+\cdots+\alpha_{m}=k}\left(u_{2}^{\alpha_{2}} \cdots u_{m}^{\alpha_{m}} u_{i}:_{R} u_{1}\right) .
\end{gathered}
$$

In addition, for $i \in \mathbb{N}$ with $2 \leq i \leq m$, one can deduce that

$$
\begin{gathered}
\left(J^{k} u_{i}:_{R} u_{1}\right)=\sum_{\alpha_{1}+\cdots+\alpha_{m}=k, \alpha_{1}>0} u_{1}^{\alpha_{1}-1} u_{2}^{\alpha_{2}} \cdots u_{m}^{\alpha_{m}} u_{i} R \\
+\sum_{\alpha_{2}+\cdots+\alpha_{m}=k} u_{2}^{\alpha_{2}} \cdots u_{m}^{\alpha_{m}}\left(u_{i}:_{R} u_{1}\right) .
\end{gathered}
$$

This implies $\sum_{i=1}^{m}\left(J^{k} u_{i}:_{R} u_{1}\right)=J^{k}$. Since $\bigcap_{j=1}^{m} \sum_{i=1}^{m}\left(J^{k} u_{i}:_{R}\right.$ $\left.u_{j}\right) \subseteq \sum_{i=1}^{m}\left(J^{k} u_{i}:_{R} u_{1}\right)$, it follows that $\left(J^{k+1}:_{R} J\right) \subseteq J^{k}$. Therefore $\left(J^{k+1}:_{R} J\right)=J^{k}$, as required.

We now turn our attention to superficial ideals, which have been introduced in [27]. In fact, let $I$ and $J$ be two ideals in a commutative Noetherian ring $S$. We say that $J$ is a superficial ideal for $I$ if the following conditions are satisfied:

(i) $G(J) \subseteq G(I)$, where $G(L)$ denotes a minimal set of generators of an ideal $L$. 
(ii) $\left(I^{k+1}:_{S} J\right)=I^{k}$ for all positive integers $k$.

On the other hand, it is easy to see that an ideal $I$ has the strong persistence property if and only if $I$ has a superficial ideal. Therefore one can replace the concept of the strong persistence property with superficial ideals. Here, we introduce a class of monomial ideals which have superficial ideals.

Theorem 3.10. (see [27, Theorem 5.10]) Suppose that I is a square-free monomial ideal in a polynomial ring $R=$ $K\left[x_{1}, \ldots, x_{n}\right]$ over a field $K$ with $G(I)=\left\{u_{1}, \ldots, u_{m}\right\}$ such that, for each $i=2, \ldots, m-1, \operatorname{gcd}\left(u_{1}, u_{2}, u_{i+1}\right)=1$ and $\left(\begin{array}{lll}u_{i}:_{R} & u_{1}\end{array}\right)$ divides $\left(u_{i+1}:_{R} u_{1}\right)$. Then I has a superficial ideal.

To illustrate Theorem 3.10, we provide the following example.

Example 3.11.(see [27, Example 5.12]) Consider the following monomial ideal

$$
\begin{aligned}
& I=\left(x_{1} x_{2} x_{3} x_{7} x_{8}, x_{1} x_{2} x_{4}, x_{3} x_{4} x_{5} x_{8}, x_{3} x_{4} x_{5} x_{6} x_{7},\right. \\
& \left.x_{4} x_{5} x_{6} x_{7} x_{8}\right),
\end{aligned}
$$

in the polynomial ring $R=K\left[x_{1}, x_{2}, x_{3}, x_{4}, x_{5}, x_{6}, x_{7}, x_{8}\right]$ over a field $K$. Now, set $u_{1}:=x_{1} x_{2} x_{3} x_{7} x_{8}, u_{2}:=$ $x_{1} x_{2} x_{4}, u_{3}:=x_{3} x_{4} x_{5} x_{8}, u_{4}:=x_{3} x_{4} x_{5} x_{6} x_{7}$, and $u_{5}:=$ $x_{4} x_{5} x_{6} x_{7} x_{8}$. It is routine to check that, for each $i=2,3,4$, $\operatorname{gcd}\left(u_{1}, u_{2}, u_{i+1}\right)=1$ and $\left(u_{i}:_{R} u_{1}\right)$ divides $\left(u_{i+1}:_{R} u_{1}\right)$. Now, Theorem 3.10 yields that the monomial ideal $\left(u_{1}, u_{2}\right)=$ $\left(x_{1} x_{2} x_{3} x_{7} x_{8}, x_{1} x_{2} x_{4}\right)$ is a superficial ideal for $I$.

We continue this argument with an elegant result which is related to the relation between normality and the strong persistence property. To accomplish this, we first give the definition of normal ideals, and next state the main theorem.

Definition 3.12.(see [27, Definition 6.1]) Let $R$ be a ring and $I$ an ideal in $R$. An element $f \in R$ is integral over $I$, if there exists an equation

$$
f^{k}+c_{1} f^{k-1}+\cdots+c_{k-1} f+c_{k}=0 \text { with } c_{i} \in I^{i} .
$$

The set of elements $\bar{I}$ in $R$ which are integral over $I$ is the integral closure of $I$. The ideal $I$ is integrally closed, if $I=\bar{I}$, and $I$ is normal if all powers of $I$ are integrally closed.

Theorem 3.13. (see [27, Theorem 6.2]) Every normal monomial ideal has the strong persistence property.

We conclude this section by exploring the strong persistence property for the cover ideals of some imperfect graphs. In fact, according to [13], the cover ideals of perfect graphs have the persistence property, but little is known for the cover ideals of imperfect graphs. More recently, it has been shown in [28] that the cover ideals of the following imperfect graphs satisfy the strong persistence property:

(1) Cycle graphs of odd orders,

(2) Wheel graphs of even orders,

(3) Helm graphs of odd orders with greater than or equal to 5.

\section{Normally Torsion-freeness for Monomial Ideals}

We first recall that an ideal $I$ in a commutative Noetherian ring $S$ is called normally torsion-free if $\operatorname{Ass}_{S}\left(S / I^{k}\right) \subseteq$ $\operatorname{Ass}_{S}(S / I)$ for all $k \in \mathbb{N}$.

The subsequent lemma guarantees that any power of a normally torsion-free square-free monomial ideal, is also normally torsion-free. Lemma 4.1. (see [29, Lemma 2.2]) Let $I$ be a square-free monomial ideal in a polynomial ring $R=K\left[x_{1}, \ldots, x_{n}\right]$. If I is normally torsion-free, then, for all positive integers $s, I^{s}$ is normally torsion-free.

In the following theorem, we introduce a class of monomial ideals which are normally torsion-free.

Theorem 4.2.(see [22, Theorem 3.3]) Let $T$ be a rooted starlike tree on the vertex set $\{z, 1, \ldots, n\}$ with root $z$. Let I be the monomial ideal corresponding to $T$ which is generated by the paths of maximal lengths such that every path is naturally oriented away from $z$, and corresponding Alexander dual $J$. Then the ideal $J$ is normally torsion-free.

As an immediate consequence of Theorem 4.2, we obtain the following corollary.

Corollary 4.3.(see [22, Corollary 3.4]) Suppose that $T$ is a rooted symmetric starlike tree on the vertex set $V(T)=$ $\{z, 1, \ldots, n\}$ with root $z$ such that every path is naturally oriented away from $z$, and the following edge set

$$
\begin{array}{r}
E(T)=\{(z, i),(k j+i, k j+k+i) \mid i=1, \ldots, k \\
\text { and } j=0, \ldots, m-1\}
\end{array}
$$

such that $n=k(m+1)$ for some $k \in \mathbb{N}$ and $m \in \mathbb{N}_{0}$. Suppose also that

$$
J_{m+1}:=\bigcap_{i=1}^{k}\left(x_{z}, x_{i}, x_{k+i}, \ldots, x_{m k+i}\right) .
$$

Then $J_{m+1}$ is normally torsion-free.

In order to demonstrate Theorem 4.7, one requires the following theorem.

Theorem 4.4.(see [29, Theorem 3.3]) Let I be a monomial ideal of $R$. Then I is normally torsion-free if and only if $I^{*}$ is.

The subsequent definition is essential for us to understand Theorem 4.7.

Definition 4.5.(see [22, Definition 3.6]) An $\left(k_{1}, k_{2}, \ldots, k_{r}\right)$ banana tree is a graph obtained by connecting one leaf of each of an $k_{i}$-star graph, for all $i=1, \ldots, r$, with a single root vertex that is distinct from all the stars.

It should be observed that, for all $i=1, \ldots, r$, the number $k_{i}$ in the definition of an $\left(k_{1}, k_{2}, \ldots, k_{r}\right)$-banana tree refers to the total number of vertices in the associated star graph. Furthermore, it is necessary to note that when $k_{1}=\cdots=$ $k_{r}=k$, we get an $(r, k)$-banana tree, as defined by Chen et al. [30].

Here, we state two examples which illustrate our definitions.

Examples 4.6.(see [22, Examples 3.7])(i) Suppose that $T$ is the tree which is shown in figure below. One can easily see 
that $T$ is a $(4,5,7)$-banana tree.

(ii) Assume that $T$ is the tree which is shown in figure below. It is routine to check that $T$ is a $(3,5)$-banana tree.

We are now ready to prove another main result in this section. Theorem 4.7. (see [22, Theorem 3.8]) Suppose that $T$ is a rooted $\left(k_{1}, k_{2}, \ldots, k_{r}\right)$-banana tree on the vertex set $V(T)=\left\{i \in \mathbb{N}: i=1,2, \ldots, k_{1}+k_{2}+\cdots+k_{r}+1\right\}$ with vertex 1 chosen as root, $s_{0}:=0, s_{i}:=\sum_{t=1}^{i} k_{t}$, and the edge set $E(T)$ is given by

$$
\begin{aligned}
& \left\{\left(1, s_{i}+2\right),\left(s_{i}+2, s_{i}+3\right),\left(s_{i}+3, s_{i}+j\right):\right. \\
& \left.i=0,1, \ldots, r-1 \text { and } j=4,5, \ldots, k_{i+1}+1\right\} .
\end{aligned}
$$

Suppose also that

$$
\begin{array}{r}
I_{2}:=\left(x_{1} x_{s_{i}+2} x_{s_{i}+3}, x_{s_{i}+2} x_{s_{i}+3} x_{s_{i}+j}:\right. \\
\left.i=0,1, \ldots, r-1 \text { and } j=4,5, \ldots, k_{i+1}+1\right\},
\end{array}
$$

and $J_{2}$ is the Alexander dual of $I_{2}$. Then the ideal $J_{2}$ is normally torsion-free.

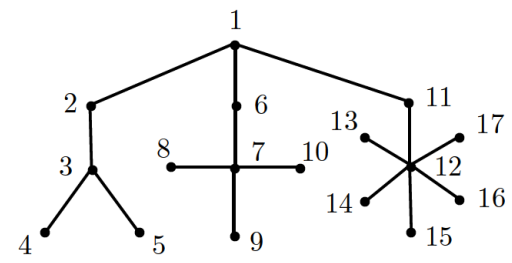

Figure 5. (4,5,7)-banana tree.

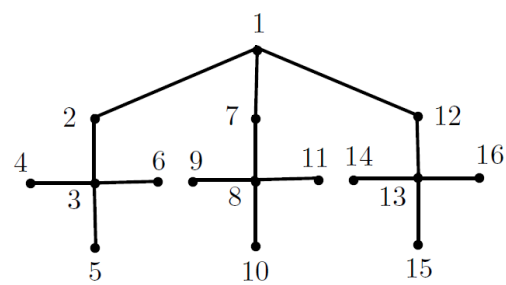

Figure 6. (3,5)-banana tree.

Proof. It follows from the hypothesis that

$J_{2}=\bigcap_{i=0}^{r-1} \bigcap_{j=4}^{k_{i+1}+1}\left(\left(x_{1}, x_{s_{i}+2}, x_{s_{i}+3}\right) \cap\left(x_{s_{i}+2}, x_{s_{i}+3}, x_{s_{i}+j}\right)\right)$

Suppose that $R=K\left[x_{i}: 1 \leq i \leq \sum_{t=1}^{r} k_{t}+1\right]$ and set $\mathfrak{q}_{i}:=\left(x_{s_{i}+2}, x_{s_{i}+3}\right)$ for all $i=0,1, \ldots, r-1$. Thus $J_{2}=\bigcap_{i=0}^{r-1}\left(\mathfrak{q}_{i}+x_{1} \prod_{j=4}^{k_{i+1}+1} x_{s_{i}+j} R\right)$. Now, put $\mathfrak{p}_{1}:=x_{1} R$, $\mathfrak{p}_{s_{i}+2}:=\mathfrak{q}_{i}$ for all $i=0,1, \ldots, r-1$, and $\mathfrak{p}_{s_{i}+j}:=x_{s_{i}+j} R$ for all $i=0,1, \ldots, r-1$ and $j=4,5, \ldots, k_{i+1}+1$. One can deduce that

$$
\begin{aligned}
J_{2} & =\bigcap_{i=0}^{r-1}\left(\mathfrak{p}_{s_{i}+2}+\mathfrak{p}_{1} \prod_{j=4}^{k_{i+1}+1} \mathfrak{p}_{s_{i}+j}\right) \\
& \left\{s_{i}+2: i=0,1, \ldots, r-1\right\} \cup\left\{1, s_{i}+j: i=0,1, \ldots, r-1 \text { and } j=4,5, \ldots, k_{i+1}+1\right\},
\end{aligned}
$$

and the following edge set $E(G)$

$$
\left\{\left\{x_{s_{i}+2}, x_{1}\right\},\left\{x_{s_{i}+2}, x_{s_{i}+j}\right\}: i=0,1, \ldots, r-1 \text { and } j=4,5, \ldots, k_{i+1}+1\right\} .
$$

Since

$$
\bigcap_{i=0}^{r-1}\left(x_{s_{i}+2} R+x_{1} \prod_{j=4}^{k_{i+1}+1} x_{s_{i}+j} R\right)=\bigcap_{i=0}^{r-1}\left(x_{s_{i}+2}, x_{1}\right) \cap \bigcap_{i=0}^{r-1} \bigcap_{j=4}^{k_{i+1}+1}\left(x_{s_{i}+2}, x_{s_{i}+j}\right),
$$


One has $G=(V(G), E(G))$ is a bipartite graph such that $F$ is the cover ideal of $G$. On the other hand, according to [15, Corollary 2.6], it follows that $F$ is normally torsion-free, and Theorem 4.4 implies that the ideal $J_{2}$ is also normally torsionfree, as claimed.

More recently, by using the cover ideals of hypergraphs and monomial localization of monomial ideals with respect to monomial prime ideals, it has been shown the following theorem.

Theorem 4.8.(see [31, Theorem 3.2]) Let T be a rooted tree. Then $I_{2}(T)^{\vee}$ is normally torsion-free.

We continue this argument with a notable result which is devoted to the relation between normally torsion-freeness and the strong persistence property.

Theorem 4.9.(see [27, Theorem 6.10]) Every normally torsion-free square-free monomial ideal has the strong persistence property.

In the sequel, we introduce four methods for constructing new classes of monomial ideals which have normally torsionfreeness. For this purpose, we begin with the first one.

Definition 4.10.(see [29, Definition 3.4]) A weight over a polynomial ring $R=K\left[x_{1}, \ldots, x_{n}\right]$ is a function $W$ : $\left\{x_{1}, \ldots, x_{n}\right\} \rightarrow \mathbb{N}$, and $w_{i}=W\left(x_{i}\right)$ is called the weight of the variable $x_{i}$. Given a monomial ideal $I$ and a weight $W$, we define the weighted ideal, denoted by $I_{W}$, to be the ideal generated by $\{h(u): u \in G(I)\}$, where $h$ is the unique homomorphism $h: R \rightarrow R$ given by $h\left(x_{i}\right)=x_{i}^{w_{i}}$. For a monomial $u \in R$, we denote $h(u)=u_{W}$.

Example 4.11. Consider the monomial ideal $I=\left(x_{1}^{2} x_{2} x_{3}^{6}, x_{2}^{3} x_{4} x_{5}^{4}\right)$ in the polynomial ring $R=K\left[x_{1}, x_{2}, x_{3}, x_{4}, x_{5}\right]$. Furthermore, let $W$ : $\left\{x_{1}, x_{2}, x_{3}, x_{4}, x_{5}\right\} \rightarrow \mathbb{N}$ be a weight over $R$ with $W\left(x_{1}\right)=2$, $W\left(x_{2}\right)=4, W\left(x_{3}\right)=2, W\left(x_{4}\right)=3$, and $W\left(x_{5}\right)=1$. Thus, the weighted ideal $I_{W}$ is given by $I_{W}=\left(x_{1}^{4} x_{2}^{4} x_{3}^{12}, x_{2}^{12} x_{4}^{3} x_{5}^{4}\right)$.

The theorem below tells us that a monomial ideal is normally torsion-free if and only if its weighted is normally torsion-free.

Theorem 4.12. (see [29, Theorem 3.10]) Let $I$ be a monomial ideal of $R$, and $W$ a weight over $R$. Then $I$ is normally torsion-free if and only if $I_{W}$ is.

We are ready to state the second method. Indeed, the following lemma says that a monomial ideal is normally torsion-free if and only if its monomial multiple is normally torsion-free.

Lemma 4.13. (see [29, Lemma 3.12]) Let I be a monomial ideal in a polynomial ring $R=K\left[x_{1}, \ldots, x_{n}\right]$ with $G(I)=$ $\left\{u_{1}, \ldots, u_{m}\right\}$, and $h=x_{j_{1}}^{b_{1}} \cdots x_{j_{s}}^{b_{s}}$ with $j_{1}, \ldots, j_{s} \in$ $\{1, \ldots, n\}$ be a monomial in $R$. Then I is normally torsionfree if and only if $h I$ is normally torsion-free.

In order to provide the third method, one should recall the definition of the monomial localization of a monomial ideal with respect to a monomial prime ideal as has been introduced in [32]. Let $I$ be a monomial ideal in a polynomial ring $R=K\left[x_{1}, \ldots, x_{n}\right]$ over a field $K$. We also denote by $V^{*}(I)$ the set of monomial prime ideals containing $I$. Let $\mathfrak{p}=\left(x_{i_{1}}, \ldots, x_{i_{r}}\right)$ be a monomial prime ideal. The monomial localization of $I$ with respect to $\mathfrak{p}$, denoted by $I(\mathfrak{p})$, is the ideal in the polynomial ring $R(\mathfrak{p})=K\left[x_{i_{1}}, \ldots, x_{i_{r}}\right]$ which is obtained from $I$ by applying the $K$-algebra homomorphism $R \rightarrow R(\mathfrak{p})$ with $x_{j} \mapsto 1$ for all $x_{j} \notin\left\{x_{i_{1}}, \ldots, x_{i_{r}}\right\}$.

We are now in a position to state the third method in the following theorem. Theorem 4.14. (see [29, Theorem 3.15]) Let $I$ be a monomial ideal in a polynomial ring $R=$ $K\left[x_{1}, \ldots, x_{n}\right]$, and $\mathfrak{p} \in V^{*}(I)$. If $I$ is normally torsion-free, then $I(\mathfrak{p})$ is so.

To express the fourth method, one requires the definition of the deletion operator, as has been given in [33, P. 303]. Let $I$ be a monomial ideal in $R=K\left[x_{1}, \ldots, x_{n}\right]$ with $G(I)=$ $\left\{u_{1}, \ldots, u_{m}\right\}$. For some $1 \leq j \leq n$, the deletion $I \backslash x_{j}$ is formed by putting $x_{j}=0$ in $u_{i}$ for each $i=1, \ldots, m$.

We finish this section with giving the fourth method in the next theorem.

Theorem 4.15. (see [29, Theorem 3.21]) Let I be a squarefree monomial ideal in $R=K\left[x_{1}, \ldots, x_{n}\right]$, and $1 \leq j \leq n$. If $I$ is normally torsion-free, then $I \backslash x_{j}$ is so.

\section{Future Works}

Several questions arise along these arguments for future works. We terminate this paper with some open questions which are devoted to the persistence property, strong persistence property, normally torsion-freeness of monomial ideals, and the unique homogeneous maximal ideal $\mathfrak{m}=$ $\left(x_{1}, \ldots, x_{n}\right)$ of $R=K\left[x_{1}, \ldots, x_{n}\right]$.

Let $I$ be a square-free monomial ideal in a polynomial ring $R=K\left[x_{1}, \ldots, x_{n}\right]$ over a field $K$, and $\mathfrak{m}=\left(x_{1}, \ldots, x_{n}\right)$ be the unique homogeneous maximal ideal of $R$. Also let $\operatorname{Ass}_{R}\left(R / I^{k}\right)=\operatorname{Ass}_{R}(R / I) \cup\{\mathfrak{m}\}$ for all $k \geq 2$. Then can we conclude that $I$ has the strong persistence property?

Let $I$ be a monomial ideal in a polynomial ring $R=$ $K\left[x_{1}, \ldots, x_{n}\right]$ and $\mathfrak{m}=\left(x_{1}, \ldots, x_{n}\right)$ be the graded maximal ideal of $R$. Then, provide a necessary and sufficient condition whether $\mathfrak{m} \in \operatorname{Ass}_{R}\left(R / I^{k}\right)$ for some positive integer $k$.

Suppose that $I$ is a square-free monomial ideal in $R=$ $K\left[x_{1}, \ldots, x_{n}\right], G(I)=\left\{u_{1}, \ldots, u_{m}\right\}, \bigcup_{i=1}^{m} \operatorname{Supp}\left(u_{i}\right)=$ $\left\{x_{1}, \ldots, x_{n}\right\}$, and $\mathfrak{m}=\left(x_{1}, \ldots, x_{n}\right)$ is the graded maximal ideal of $R$. If there exists a positive integer $1 \leq j \leq n$ such that $\mathfrak{m} \backslash x_{j} \in \operatorname{Ass}_{R \backslash x_{j}}\left(\left(R \backslash x_{j}\right) /\left(I \backslash x_{j}\right)^{k}\right)$ for some positive integer $k$, then can we deduce that $\mathfrak{m} \in \operatorname{Ass}_{R}\left(R / I^{k}\right)$ ? 5

To understand the subsequent questions, we recall the definition of polarization. (see [33, Definition 4.1]) The process of polarization replaces a power $x_{i}^{t}$ by a product of $t$ new variables $x_{(i, 1)} \cdots x_{(i, t)}$. We call $x_{(i, j)}$ a shadow of $x_{i}$. We will use $\widetilde{I}^{t}$ to denote the polarization of $I^{t}$, will use $S_{t}$ for the new polynomial ring in this polarization, and will use $\widetilde{w}$ to denote the polarization in $S_{t}$ of a monomial $w$ in $R=K\left[x_{1}, \ldots, x_{n}\right]$. The depolarization of an ideal in $S_{t}$ is formed by setting $x_{(i, j)}=x_{i}$ for all $i, j$.

Let $I$ be a normally torsion-free non-square-free monomial ideal in a polynomial ring $R=K\left[x_{1}, \ldots, x_{n}\right]$. Then can we deduce that $\widetilde{I}$ is a normally torsion-free monomial ideal? 
Let $I$ and $J$ be two monomial ideals in $R=K\left[x_{1}, \ldots, x_{n}\right]$. If $J$ is a superficial ideal for $I$, then is $\widetilde{J}$ a superficial ideal for $\widetilde{I}$ ?

\section{Conclusion}

In general, investigating the persistence property, strong persistence property, and normally torsion-freeness of monomial ideals have been of interest for many researchers. Connecting to graph theory, path ideals, edge ideals, and cover ideals of certain graphs are shown to have these properties, but finding the other classes of monomial ideals with these properties is going on. In this paper, we try to collect the latest results in this field. Also, it is very interesting to find the other applications of these notions in combinatorics as it has been shown in [13], persistence property has some applications in colorings of graph.

\section{Acknowledgements}

The author is deeply grateful to anonymous referee for careful reading of the manuscript, and for his/her valuable suggestions which led to several improvements in the quality of this paper.

\section{References}

[1] Brodmann M. Asymptotic stability of $\operatorname{Ass}\left(M / I^{n} M\right)$. Proc. Amer. Math. Soc. 1979; 74:16-18. doi: 10.2307/2042097.

[2] Hoa L. T. Stability of associated primes of monomial ideals. Vietnam J. Math. 2006; 34: 473-487.

[3] Khashyarmanesh K, Nasernejad M. On the stable set of associated prime ideals of monomial ideals and squarefree monomial ideals. Comm. Algebra. 2014; 42: $3753-$ 3759. doi: 10.1080/00927872.2013.793696.

[4] Khashyarmanesh K, Nasernejad M. Some results on the associated primes of monomial ideals. Southeast Asian Bull. Math. 2015; 39: 439-451.

[5] McAdam S. Asymptotic Prime Divisors. Lecture Notes in Mathematics. 103. New York: Springer-Verlag; 1983. $120 \mathrm{p}$.

[6] Herzog J, Hibi T. The depth of powers of an ideal. J. Algebra 2005; 291: 534-550. doi: 10.1016/j.jalgebra.2005.04.007.

[7] Martínez-Bernal J, Morey S, Villarreal R. H. Associated primes of powers of edge ideals. J. Collect. Math. 2012; 63: 361-374. doi: 10.1007/s13348-011-0045-9.

[8] Morey S, Villarreal R. H. Edge ideals: Algebraic and combinatorial properties, Progress in commutative Algebra. 2012; 1: 85-126.
[9] Kaiser T, Stehlík M, Škrekovski R. Replication in critical graphs and the persistence of monomial ideals. J. Comb. Theory Ser. A. 2014; 123: 239-251. doi: 10.1016/j.jcta.2013.12.005.

[10] Ratliff L. J. Jr. On prime divisors of $I^{n}, n$ large. Michigan Math. J. 1976; 23: 337-352. doi: $10.1307 / \mathrm{mmj} / 1029001769$.

[11] Reyes E, Toledo J. On the strong persistence property for monomial ideals. Bull. Math. Soc. Sci. Math. Roumanie (N.S.) 2017; 60(108): 293-305.

[12] Herzog J, Rauf A, Vladoiu M. The stable set of associated prime ideals of a polymatroidal ideal. J. Algebraic Combin. 2013; 37: 289-312. doi: 10.1007/s10801-0120367-z.

[13] Francisco C. A, Hà H. T, Van Tuyl A. Colorings of hypergraphs, perfect graphs and associated primes of powers of monomial ideals. J. Algebra. 2011; 331: 224242. doi: 10.1016/j.jalgebra.2010.10.025.

[14] Simis A, Vasconcelos W, Villarreal R. H. On the ideal theory of graphs. J. Algebra. 1994; 167: 389-416. doi: 10.1006/jabr.1994.1192.

[15] Gitler I, Reyes E, Villarreal R. H. Blowup algebras of ideals of vertex covers of bipartite graphs. Contemp. Math. 2005; 376: 273-279.

[16] Hungerford T. W. Algebra,. Graduate Texts in Mathematics. 73. New York: Springer-Verlag, 1974. 504 p.

[17] Nasernejad M. A new proof of the persistence property for ideals in Dedekind rings and Prüfer domains. J. Algebr. Syst. 2013; 1: 91-100. doi: 10.22044/jas.2014.229.

[18] Nasernejad M. Persistence property for associated primes of a family of ideals. Southeast Asian Bull. Math. 2016; 40: $571-575$.

[19] Villarreal R. H. Cohen-Macaulay graphs. Manuscripta Math. 1990; 66: 277-293. doi: 10.1007/BF02568497.

[20] Conca A, De Negri E. M-sequences, graph ideals, and ladder ideals of linear type. J. Algebra. 1999; 211: 599624. doi:10.1006/jabr.1998.7740.

[21] Nasernejad M. Asymptotic behaviour of assicated primes of monomial ideals with combinatorial applications. J. Algebra Relat. Topics. 2014; 2: 15-25.

[22] Nasernejad M, Khashyarmanesh K. On the Alexander dual of the path ideals of rooted and unrooted trees. Comm. Algebra. 2017; 45: 1853-1864. doi: 10.1080/00927872.2016.1226855. 
[23] Bhat A, Biermann J, Van Tuyl A. Generalized cover ideals and the persistence property. J. Pure Appl. Algebra. 2014; 218: 1683-1695. doi: 10.1016/j.jpaa.2014.01.007.

[24] Bayati S, Herzog J. Expansions of monomial ideals and multigraded modules. Rocky Mountain J. Math. 2014; 44: 1781-1804. doi: 10.1216/RMJ-2014-44-6-1781.

[25] Nasernejad M. Persistence property for some classes of monomial ideals of a polynomial ring. J. Algebra Appl. 2017; 16: 1750105 (17 pages). doi: $10.1142 / \mathrm{S} 0219498817501055$.

[26] Nasernejad M, Rajaee S. Detecting the maximalassociated prime ideal of monomial ideals. Bol. Soc. Mat. Mex. doi: 10.1007/s40590-018-0208-8.

[27] Rajaee S, Nasernejad M, Al-Ayyoub I. Superficial ideals for monomial ideals. J. Algebra Appl. 2018; 17: 1850102 (28 pages). doi: 10.1142/S0219498818501025.

[28] Nasernejad M, Khashyarmanesh K, Al-Ayyoub I. Associated primes of powers of cover ideals under graph operations. Comm. Algebra. doi: 10.1080/00927872.2018.1527920.

[29] Sayedsadeghi M, Nasernejad M. Normally torsionfreeness of monomial ideals under monomial operators. Comm. Algebra. doi: 10.1080/00927872.2018.1469029.

[30] Chen W. C, Lu H. I, and Yeh Y. N. Operations of Interlaced Trees and Graceful Trees. Southeast Asian Bull. Math. 1997; 21: 337-348.

[31] Khashyarmanesh K, Nasernejad M. A note on the Alexander dual of path ideals of rooted trees. Comm. Algebra. 2018; 46: 283-289. doi: 10.1080/00927872.2017.1324867.

[32] Herzog J, Qureshi A. A. Persistence and stability properties of powers of ideals. J. Pure Appl. Algebra. 2015; 219: 530-542. doi: 10.1016/j.jpaa.2014.05.011.

[33] Hà H. T, Morey S. Embedded associated primes of powers of square-free monomial ideals. J. Pure Appl. Algebra. 2010; 214: 301-308. doi: 10.1016/j.jpaa.2009.05.002. 